\title{
Modeling and Identification of Discrete-Time Nonlinear Dynamic Cascade Systems with Input Hysteresis
}

\author{
Jozef Vörös \\ Faculty of Electrical Engineering and Information Technology, Slovak Technical University, Ilkovicova 3, 81219 Bratislava, Slovakia \\ Correspondence should be addressed to Jozef Vörös; jozef.voros@stuba.sk
}

Received 22 February 2015; Accepted 29 April 2015

Academic Editor: Hiroyuki Mino

Copyright (C) 2015 Jozef Vörös. This is an open access article distributed under the Creative Commons Attribution License, which permits unrestricted use, distribution, and reproduction in any medium, provided the original work is properly cited.

A simple approach to modeling and identification of discrete-time nonlinear dynamic systems having an input hysteresis in cascade with a linear dynamic system is presented. A special form of Coleman-Hodgdon model for the hysteresis is considered, which is linear in parameters. For the cascade system parameter estimation, an iterative method with internal variable estimation is proposed. Simulation studies of cascade systems identification using special periodic inputs are included.

\section{Introduction}

Hysteresis is a special type of multivalued nondifferentiable nonlinearity and is encountered in a variety of processes where memory effects are involved between the input and output variables. The hysteresis is a dynamic nonlinearity, because the current output depends also on the history of the input $[1,2]$. It can be found in biology, optics, electronics, ferroelectricity, magnetism, mechanics, and so forth.

In many control applications, the presence of the hysteretic behavior in sensors and actuators causes a hard nonlinear relationship between inputs and outputs. This phenomenon occurs in all the smart material-based actuators such as piezoceramics, magnetostrictive, and shape memory alloys [3-5]. For example, the piezoelectric devices are widely applied to the positioning systems because of their high positioning accuracy, large driving forces, and rapid response capability [6]. However, the existence of hysteresis often limits their performance. The hysteresis in cascade with linear dynamic systems can lead to instability in closedloop operations and complicates the task of controller design and analysis [7]. Therefore, modeling and identification of such nonlinear cascade systems is a significant problem that should be solved for the application of these devices.

It is of great importance to find the best models approximating the systems with hysteretic nonlinearities. To describe the behavior of hysteretic processes, several mathematical models have been suggested and a survey may be found in [8]. In addition, more approaches have been proposed for the identification and control of different types of hysteretic systems; see, for example, [9-13].

For a broad class of hysteretic systems, a first-order scalar time-domain differential equation can be used to describe the system behavior [14]. A relatively simple differential model of hysteresis, which is appropriate for the representation of rate independent hysteretic systems, is the so-called ColemanHodgdon model studied in [15-17]. This model is able to capture, in an analytical form, a range of shapes of hysteretic loops, which match the behavior of a wide class of hysteretic systems. Applications of this differential equation model based on more or less complex solutions of the differential equation were used, for example, in [6, 18-22].

In this paper, a new and simple approach to modeling and identification of discrete-time cascade systems with an input hysteresis followed by a linear dynamic system is presented. It means that the discussed system is a cascade of nonlinear dynamic and linear dynamic subsystems. A special form of the Coleman-Hodgdon model is considered for the hysteresis. This is based on using piecewise-linear "material functions," introducing an appropriate internal variable, and rearranging the model equation. The resulting description of the whole cascade system is linear in parameters. For the cascade model parameter estimation, an iterative method with internal variable estimation is proposed. This enables performing 
the identification of cascade systems with input hysteresis on the basis of available input/output data. Simulation studies of cascade systems identification using special periodic inputs are included to demonstrate the feasibility of proposed approach. To the author's knowledge, no work dealing with this problem was published up to now.

\section{Coleman-Hodgdon Hysteresis Model}

The differential model of hysteresis (the so-called Duham model) is a representation of a rate-independent dynamic effect in the form of the first-order nonlinear differential equation in the time domain

$$
\dot{x}=F(u, x)|\dot{u}|+G(u, x) \dot{u},
$$

where $x$ is the output and $u$ is the input. Both $x$ and $u$ are real-valued functions of time with piecewise continuous derivatives $\dot{x}$ and $\dot{u}$. In the following, the Coleman-Hodgdon model of hysteresis, based on function $F(u, x)$ that is affine in $x$ and function $G(u, x)$ that is constant in $x$ will be considered. Then the hysteresis model can be written as

$$
\dot{x}=\alpha[f(u)-x]|\dot{u}|+g(u) \dot{u}
$$

with $\alpha>0$ being constant. It is assumed that

(i) the real-valued function $f(\cdot)$ is odd, is monotonely increasing, and is piecewise continuously differentiable with a finite limit for its first-order derivative at positive infinity;

(ii) the real-valued function $g(\cdot)$ is even and piecewise continuous and at infinity of such a finite value that

$$
\lim _{s \rightarrow \infty} \frac{d f(s)}{d s}=\lim _{s \rightarrow \infty} g(s) ;
$$

(iii) the real-valued functions $f(\cdot)$ and $g(\cdot)$ are such that

$$
\begin{aligned}
& \frac{d f(s)}{d s} \geq g(s), \\
& \alpha e^{\alpha s} \int_{s}^{\infty}\left[\frac{d f(\varsigma)}{d \varsigma}-g(\varsigma)\right] e^{-\alpha \varsigma} d \varsigma \leq g(s), \\
& \forall s<\infty .
\end{aligned}
$$

The solutions of differential equation (2) move on the curves given by

$$
\frac{d x}{d u}=\alpha[f(u)-x] \operatorname{sgn}(\dot{u})+g(u)
$$

and this can be transformed into the following discrete form by replacing the differentials with the corresponding differences and rearranging as follows:

$$
\begin{aligned}
x(t+1)-x(t) & \\
= & \alpha\{f[u(t)]-x(t)\}|u(t+1)-u(t)| \\
& +g[u(t)][u(t+1)-u(t)],
\end{aligned}
$$

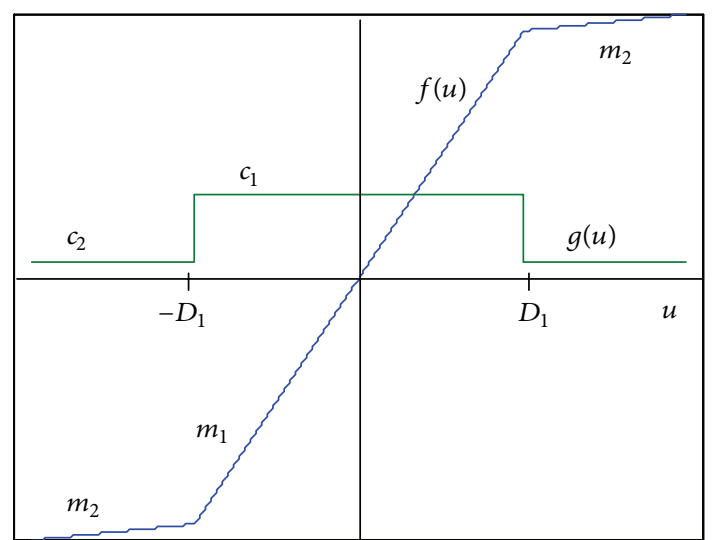

FIGURE 1: Functions $f$ and $g$.

where $t=1,2,3, \ldots$ is the discrete time. This description can be used for a broad class of hysteretic systems by an appropriate choice of the "material" functions $f(\cdot)$ and $g(\cdot)$ shaping the hysteresis. In [15], the following forms of piecewise-linear functions $f(\cdot)$ and $g(\cdot)$ were proposed (see Figure 1):

$$
\begin{aligned}
& f[u(t)] \\
& = \begin{cases}m_{2}\left[u(t)-D_{1}\right]+m_{1} D_{1}, & \text { if } D_{1}<u(t) \\
m_{1} u(t), & \text { if }-D_{1} \leq u(t) \leq D_{1} \\
m_{2}\left[u(t)+D_{1}\right]-m_{1} D_{1}, & \text { if } u(t)<-D_{1}\end{cases} \\
& g[u(t)]= \begin{cases}c_{2}, & \text { if } D_{1}<u(t) \\
c_{1}, & \text { if }-D_{1} \leq u(t) \leq D_{1} \\
c_{2}, & \text { if } u(t)<-D_{1},\end{cases}
\end{aligned}
$$

where $m_{1}, m_{2}$ are the slopes of $f(\cdot)$ and the constant $D_{1}>0$ determines the range of the central segment of $f(\cdot)$ as well as that of $g(\cdot)$ with the constant values $c_{1}>c_{2}$. Evidently, the functions $f(\cdot)$ and $g(\cdot)$ agree with conditions (i)-(iii) and the major hysteresis loop and some minor loops generated by hysteresis model (6) based on these functions using triangular inputs $u(t)$ with different amplitudes are shown in Figure 2. Note that the parts of the graph connecting the loops are not plotted to improve the visibility of loops.

This model can be simplified in the case of hysteresis with assumed major loop saturation, when the values of $m_{2}$ and $c_{2}$ are near zero and can be neglected [18]. Then the hysteresis model is characterized by only 4 parameters: $\alpha, m_{1}, D_{1}$, and $c_{1}$. However, estimating the model parameters on the basis of inputs $u(t)$ and outputs $x(t)$ is not easy because of the complicated mathematical model description. One way how to solve this problem is to consider the so-called loading and unloading forms of model equation by substituting the corresponding segments of $f(\cdot)$ and $g(\cdot)$ from (7) into (6).

Another way is to consider appropriate analytical forms of (7) enabling solving the problem of hysteresis model 


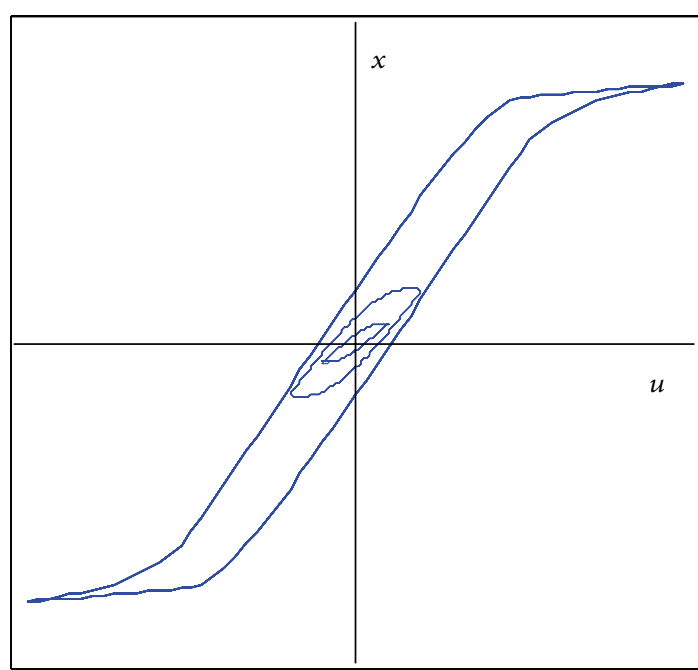

Figure 2: Hysteresis.

parameter estimation as a pseudolinear one [23]. Using the switching function defined as

$$
h(\tau)= \begin{cases}0, & \text { if } \tau>0 \\ 1, & \text { if } \tau \leq 0\end{cases}
$$

functions (7) can be rewritten as follows:

$$
\begin{aligned}
f[u(t)]= & m_{1} u(t)\left[1-h\left(D_{1}-|u(t)|\right)\right] \\
& +D_{1} m_{1} h\left(D_{1}-|u(t)|\right) \operatorname{sign}[u(t)], \\
g[u(t)]= & c_{1}\left[1-h\left(D_{1}-|u(t)|\right)\right] .
\end{aligned}
$$

After substituting (9) into (6), the hysteresis model will be linear in the parameters $\alpha$ and $c_{1}$, and in the products of parameters $\alpha m_{1}$ and $\alpha m_{1} D_{1}$, but the parameter $D_{1}$ appears also nonlinearly in the switching function. Defining the following internal variable

$$
\xi(t)=h\left[D_{1}-|u(t)|\right]
$$

and assigning

$$
\begin{aligned}
& m=\alpha m_{1}, \\
& D=\alpha m_{1} D_{1},
\end{aligned}
$$

the model equation can be written as

$$
\begin{aligned}
x(t+1)= & x(t)-\alpha x(t)|u(t+1)-u(t)| \\
& +m u(t)[1-\xi(t)]|u(t+1)-u(t)| \\
& +D \xi(t) \operatorname{sign}[u(t)]|u(t+1)-u(t)| \\
& +c_{1}[1-\xi(t)][u(t+1)-u(t)] .
\end{aligned}
$$

Now the hysteresis model equation is in the form where all the model parameters are separated. Note that similarly we can find the descriptions of $f(\cdot)$ and $g(\cdot)$ for nonzero values of $m_{2}$ and $c_{2}$. Then the model will have 6 parameters plus one more internal variable.

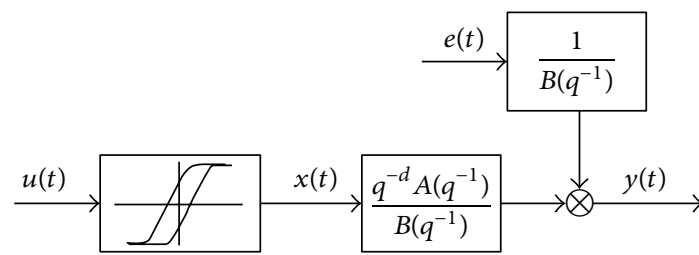

FIgURE 3: Cascade model with input hysteresis.

\section{Cascade System with Input Hysteresis}

Cascade systems consist of serially connected linear and nonlinear subsystems. One of the simplest cases is the connection of a static nonlinear subsystem followed by a linear dynamic one. This cascade system is known as the Hammerstein system and there are lots of identification methods for different types of nonlinearities and corresponding models. For example, many identification methods have been presented for the Hammerstein systems such as different iterative algorithms [24-26], or gradient based algorithms $[27,28]$. Some approaches deal with special types of nonlinearities such as saturation and dead-zone [2931], piecewise-linear [32-34], or discontinuous [35]. The parameter estimation problems are often solved using the key term separation principle [36-38] and generalized or control oriented approaches are considered in $[39,40]$. Techniques based on nonuniform sampling or oversampling are presented in $[41,42]$ and nonparametric or combined parametric-nonparametric identification is presented in [43, 44]. In addition, some specific methods based on particle swarm optimization [45] or neural networks [46] can be applied for identification of Hammerstein systems.

However, in many real control systems, the hysteresis appears in a cascade connection with a linear dynamic system. One of the possible cases is the cascade system where the hysteresis is followed by a linear dynamic system as shown in Figure 3. The linear dynamic system can be described by the difference equation

$$
B\left(q^{-1}\right) y(t)=q^{-d} A\left(q^{-1}\right) x(t)+e(t),
$$

where $x(t)$ and $y(t)$ are the inputs and outputs, respectively, and $A\left(q^{-1}\right)$ and $B\left(q^{-1}\right)$ are scalar polynomials in the unit delay operator $q^{-1}$ :

$$
\begin{aligned}
& A\left(q^{-1}\right)=a_{0}+a_{1} q^{-1}+\cdots+a_{r} q^{-r}, \\
& B\left(q^{-1}\right)=1+b_{1} q^{-1}+\cdots+b_{n} q^{-n} .
\end{aligned}
$$

$d \geq 1$ is a pure delay and $e(t)$ is a white noise with zero mean.

The output equation of this cascade model can be derived from (12) and (13). However, a direct substitution of (12) into (13) would lead to a very complex expression; therefore, the so-called key term separation principle can be 
applied $[47,48]$. In this connection of two systems, we can assume that $a_{0}=1$; that is,

$$
\begin{aligned}
y(t)= & x(t-d)+\sum_{i=1}^{r} a_{i} x(t-d-i)-\sum_{j=1}^{n} b_{j} y(t-j) \\
& +e(t),
\end{aligned}
$$

and we can substitute (12) only for the separated variable $x(t-$ d) leading to the following equation:

$$
\begin{aligned}
y(t)=x( & t-d-1)-\alpha x(t-d-1) \\
& \cdot|u(t-d)-u(t-d-1)|+m u(t-d-1) \\
& \cdot[1-\xi(t-d-1)] \\
& \cdot|u(t-d)-u(t-d-1)|+D \xi(t-d-1) \\
& \cdot \operatorname{sign}[u(t-d-1)] \\
& \cdot|u(t-d)-u(t-d-1)| \\
+ & c_{1}[1-\xi(t-d-1)] \\
& \cdot[u(t-d)-u(t-d-1)] \\
+ & \sum_{i=1}^{r} a_{i} x(t-d-i)-\sum_{j=1}^{n} b_{j} y(t-j)+e(t),
\end{aligned}
$$

where the parameters of both the hysteresis and the linear system are separated and the equation is quasilinear because the internal variables $\xi(t)$ and $x(t)$ depend on the hysteresis parameters.

Defining the following vector of data

$$
\begin{aligned}
& \varphi(t)=\{-x(t-d-1)|u(t-d)-u(t-d-1)|, \\
& u(t-d-1)[1-\xi(t-d-1)] \\
& \cdot|u(t-d)-u(t-d-1)|, \xi(t-d-1) \\
& \quad \cdot \operatorname{sign}[u(t-d-1)]|u(t-d)-u(t-d-1)|, \\
& \quad[1-\xi(t-d-1)][u(t-d)-u(t-d-1)], \\
& \quad x(t-d-1), \ldots, x(t-d-r),-y(t-1), \ldots, \\
& -y(t-n)\}^{T}
\end{aligned}
$$

and the vector of parameters

$$
\theta=\left[\alpha, m, D, c_{1}, a_{1}, \ldots, a_{r}, b_{1}, \ldots, b_{n}\right]^{T}
$$

the mathematical model for the cascade system with input hysteresis can be written in the concise form:

$$
y(t)-x(t-d-1)=\varphi^{T}(t) \theta+e(t),
$$

and the model parameter estimation can be solved as a linear estimation problem.

\section{Parameter Estimation}

As the variables $\xi(t)$ and $x(t)$ are not available and must be estimated, an iterative parameter estimation process has to be considered. Assigning the estimated variables in the sth step as

$$
\begin{aligned}
{ }^{s} \xi(t)= & h\left[{ }^{s} D_{1}-|u(t)|\right]=h\left[\frac{{ }^{s} D}{{ }^{s} m}-|u(t)|\right], \\
{ }^{s} x(t+1)= & { }^{s} x(t)-{ }^{s} \alpha^{s} x(t)|u(t+1)-u(t)| \\
& +{ }^{s} m u(t)\left[1-{ }^{s} \xi(t)\right]|u(t+1)-u(t)| \\
& +{ }^{s} D^{s} \xi(t) \operatorname{sign}[u(t)]|u(t+1)-u(t)| \\
& +{ }^{s} c_{1}\left[1-{ }^{s} \xi(t)\right][u(t+1)-u(t)],
\end{aligned}
$$

the estimate of data vector as

$$
\begin{aligned}
& { }^{s} \varphi(t)=\left\{-{ }^{s} x(t-d-1)|u(t-d)-u(t-d-1)|,\right. \\
& u(t-d-1)\left[1-{ }^{s} \xi(t-d-1)\right] \\
& \cdot|u(t-d)-u(t-d-1)|,{ }^{s} \xi(t-d-1) \\
& \quad \cdot \operatorname{sign}[u(t-d-1)]|u(t-d)-u(t-d-1)|, \\
& \quad\left[1-{ }^{s} \xi(t-d-1)\right][u(t-d)-u(t-d-1)], \\
& { }^{s} x(t-d-1), \ldots,{ }^{s} x(t-d-r),-y(t-1), \ldots, \\
& -y(t-n)\}^{T},
\end{aligned}
$$

and the estimate of parameter vector

$$
{ }^{s} \theta=\left[{ }^{s} \alpha,{ }^{s} m,{ }^{s} D,{ }^{s} c_{1},{ }^{s} a_{1}, \ldots,{ }^{s} a_{r},{ }^{s} b_{1}, \ldots,{ }^{s} b_{n}\right]^{T} \text {, }
$$

the error to be minimized for the estimation procedure is

$$
{ }^{s+1} \mathcal{\varepsilon}(t)=y(t)-{ }^{s} x(t-d-1)-{ }^{s} \varphi^{T}(t){ }^{s+1} \theta,
$$

where ${ }^{s} \varphi(t)$ is the data vector with the corresponding estimates of internal variable and ${ }^{s+1} \theta$ is the $(s+1)$ th estimate of the parameter vector. If the mean squares errors criterion is used, the following functional will be minimized with respect to the parameter vector:

$$
{ }^{s+1} J=\frac{1}{N} \sum_{t=1}^{N}{ }^{s+1} \varepsilon^{2}(t),
$$

where $N$ is the number of input/output samples.

The steps in the iterative estimation procedure may be now stated as follows:

(a) Minimizing ${ }^{s+1} J$ based on (23), the estimates of parameters ${ }^{s+1} \theta$ are computed using ${ }^{s} \varphi(t)$ with the $s$ th estimates of internal variables.

(b) Using (20), the estimates of ${ }^{s+1} \varphi(t)$ are evaluated by means of the recent estimates of corresponding parameters. 
(c) If the estimation criterion is met, that is, the error is less than a predetermined value, the procedure ends; else it continues by repeating steps (a) and (b).

In the first iteration, nonzero initial values of hysteresis parameters $m$ and $D$ have to be used for computation of the first estimates of variable $\xi(t)$, and the first estimates of internal variable $x(t)$ are chosen as ${ }^{1} x(t)=u(t-1)$. It is straightforward to choose ${ }^{1} m_{1}=1.0$ and ${ }^{1} D_{1}$ close to the maximum amplitude of the input.

The identification consists in exciting the system with an input signal $p(t)$, which is continuous and periodic of period $T>0$. This class of inputs is very common in identification procedures for hysteretic systems and includes sine waves with or without offset, triangular waves, and so forth [10]. Moreover, similarly as in [11], we consider that disturbance $d(t)$ is added to the input signal $p(t)$ resulting in corrupted input signal

$$
u(t)=p(t)+d(t)
$$

to make input-output data set informative enough to guarantee uniqueness of the estimates of hysteresis parameters. The disturbance $d(t)$ can be chosen as a normally distributed random variable with zero mean and not correlated with $e(t)$. Note that it is beneficial to use a sequence of such periodic inputs $u(t)$ with different range of amplitudes for $p(t)$, as similar loops of hysteresis are repeated in each cycle of operation depending on the range of input amplitudes.

\section{Examples}

To illustrate the feasibility of proposed identification method, the following examples of simulated cascade systems with input hysteresis are presented.

Example 1. The input hysteresis described by model (12) was considered with the following parameters: $\alpha=2.0, m_{1}=1.5$, $D_{1}=0.7$, and $c_{1}=0.5$. The hysteresis loop is shown in Figure 4 . The linear dynamic system was described by the difference equation:

$$
\begin{aligned}
y(t)= & x(t-1)-0.5 x(t-2)+0.2 y(t-1) \\
& -0.35 y(t-2)
\end{aligned}
$$

The identification was performed on the basis of $N=1632$ inputs $u(t)$ and generated outputs $y(t)$. As shown in Figure 5, a $T$-periodic triangular signal $p(t)$ was chosen $(T=204)$; the peak amplitudes were $U_{1}=1.5$ for $t \in[1,2 T], U_{2}=0.5 U_{1}$ for $t \in[2 T+1,4 T], U_{3}=0.1 U_{1}$ for $t \in[4 T+1,6 T]$, and $U_{4}=0.5 U_{1}$ for $t \in[6 T+1,8 T]$. Then a uniformly distributed random disturbance with $|d(t)|<0.125 U_{1}$ was superposed on $p(t)$. Note that the different range of amplitudes for $p(t)$ was chosen to cover the major and two minor loops of the hysteresis. Normally distributed random noise with zero mean and signal-to-noise ratio $\mathrm{SNR}=50$ (the square root of the ratio of output and noise variances) was added to the generated outputs. The initial values ${ }^{1} m_{1}=1.0$ and ${ }^{1} D_{1}=$ 1.5 were used for the first estimate of $\xi(t)$. The process of

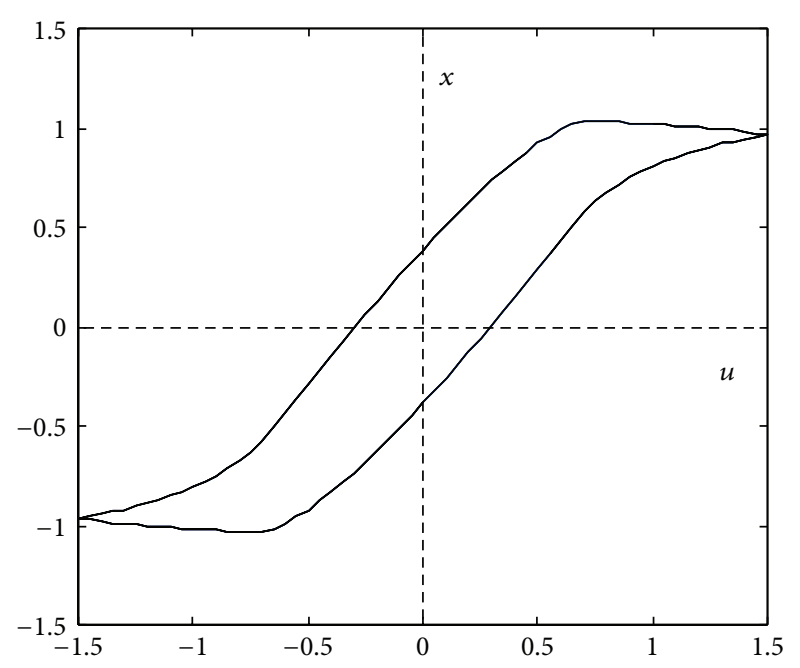

FIgURE 4: Input hysteresis: Example 1.

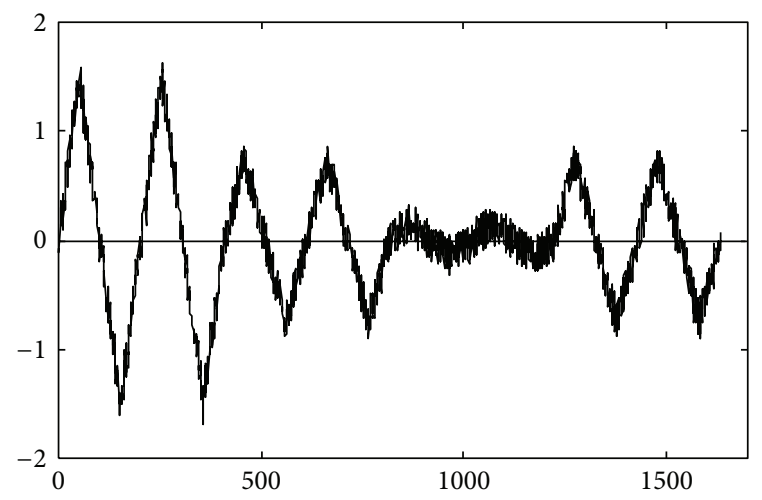

FIgURE 5: Input variable $u(t)$ : Example 1.

hysteresis parameter estimation is shown in Figure 6 (the topdown order of parameters is $\alpha, m_{1}, D_{1}$, and $c_{1}$ ), while that of linear system is shown in Figure 7 (the top-down order of parameters is $b_{2}, b_{1}$, and $\left.a_{1}\right)$. The parameter estimates reach the true values of given parameters after about 10 iterations and remain unchanged during the further iterations.

Example 2. The input hysteresis described by model (12) was considered with the following parameters: $\alpha=5.0, m_{1}=2.0$, $D_{1}=0.9$, and $c_{1}=0.7$. The hysteresis loop is shown in Figure 8 . The following linear dynamic system was described by the difference equation

$$
\begin{aligned}
y(t)= & x(t-1)+0.5 x(t-2)+0.2 y(t-1) \\
& -0.35 y(t-2) .
\end{aligned}
$$

The simulation of cascade system was performed similarly as in the above example for $U_{1}=2.0$ (see Figure 9). The initial values ${ }^{1} m_{1}=1.0$ and ${ }^{1} D_{1}=2.0$ were used for the first estimate of $\xi(t)$. The process of hysteresis parameter estimation is shown in Figure 10 (the top-down order of parameters is $\alpha, m_{1}, D_{1}$, and $c_{1}$ ), while that of linear system is shown in Figure 11 (the top-down order of parameters is 


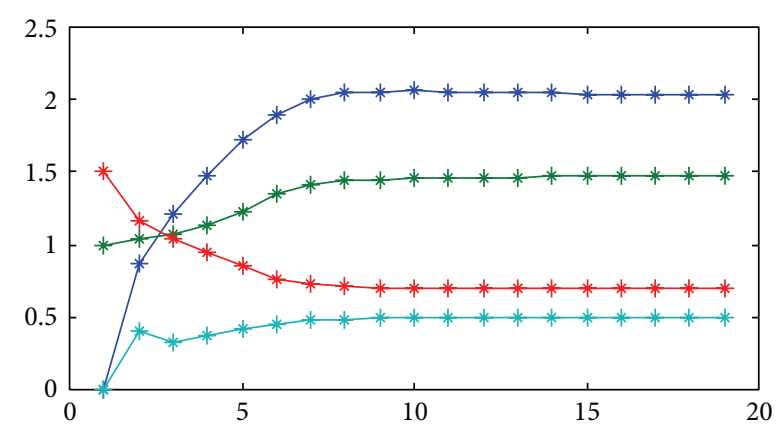

FIGURE 6: Estimates of hysteresis parameters: Example 1.

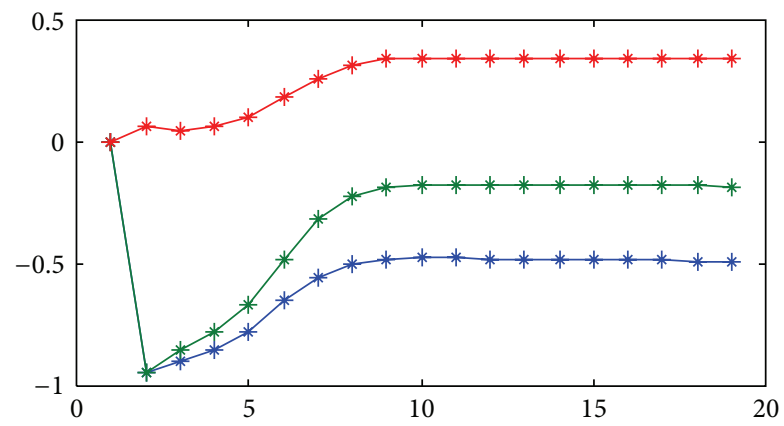

FIGURE 7: Estimates of linear system parameters: Example 1.

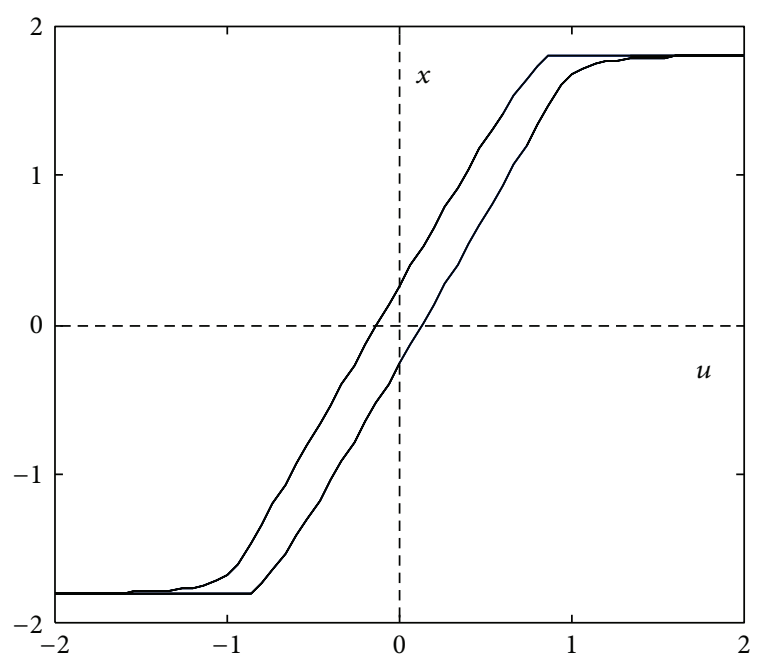

Figure 8: Input hysteresis: Example 2.

$a_{1}, b_{2}$, and $\left.b_{1}\right)$. The estimates converge to the values of given parameters after about 10 iterations.

\section{Conclusions}

To deal with the control problems of a cascade system with an input hysteresis, the characterization of the hysteresis forms the most important task. Appropriate hysteresis models may then be applied to the formulation of control algorithms. Therefore, the modeling and identification of systems with hysteresis is of great importance.

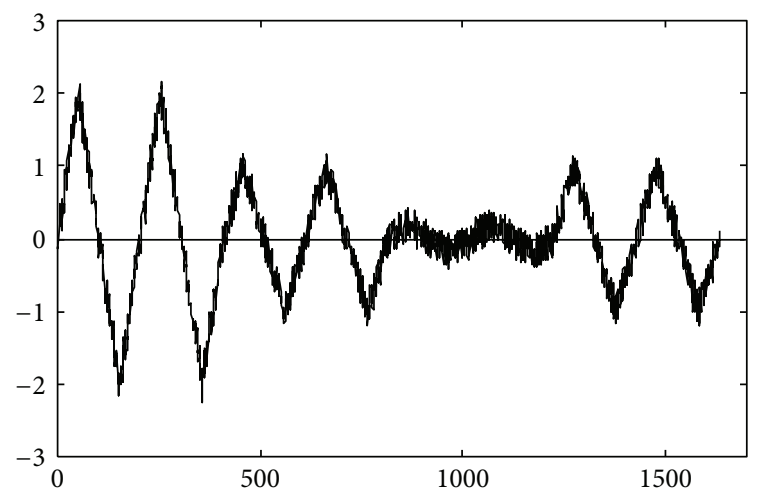

FIGURE 9: Input variable $u(t)$ : Example 2 .

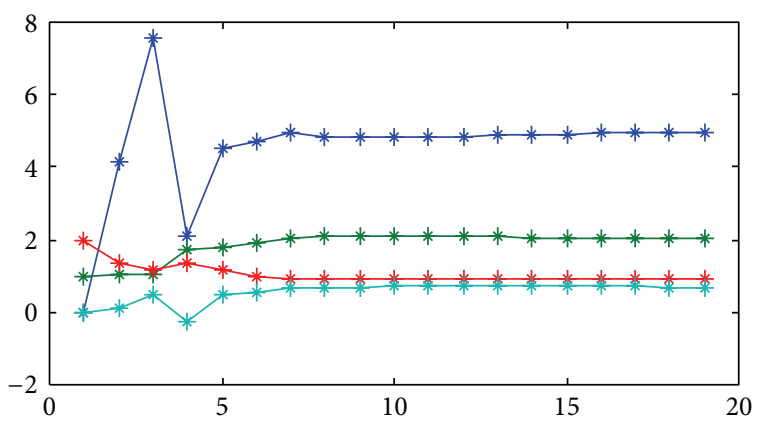

FIGURE 10: Estimates of hysteresis parameters: Example 2.

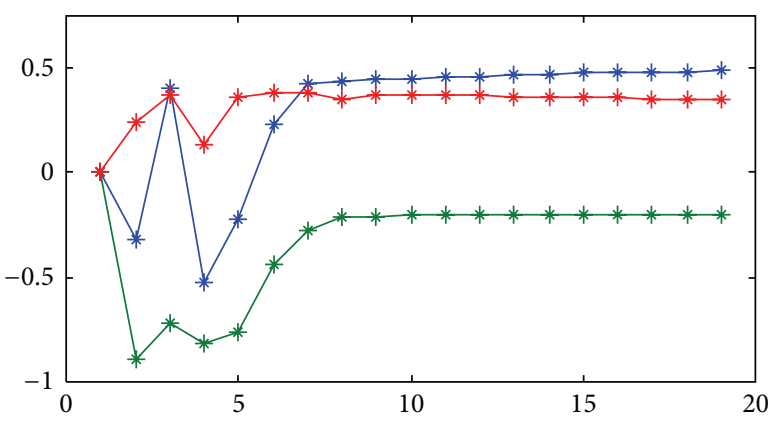

FIGURE 11: Estimates of linear system parameters: Example 2.

In this paper, a new approach to modeling and identification of nonlinear dynamic cascade systems with input hysteresis using the Coleman-Hodgdon model has been presented. The proposed model for hysteresis is based on the special form of $f(\cdot)$ and $g(\cdot)$ representations. For this cascade model, an iterative identification algorithm was proposed with internal variable estimation based on input/output data. Although a general proof of convergence for the iterative algorithm with internal variable estimation is not available $[47,48]$, the simulation results show good convergence.

The presented identification method is of an easy use and the proposed model can be applied for online identification of cascade systems with input hysteresis using the known recursive least-squares algorithm. Furthermore, no a priori knowledge on the parameters is assumed except very rough 
initial values of the parameters required for the first estimates of internal variables. The proposed mathematical model can be used also in the framework of recursive identification [49]. This approach may be a novel contribution to the solution of problems arising in many application areas dealing with the cascade systems with input hysteresis. Finally note that even more complex forms of Coleman-Hodgdon model can be considered for modeling the input hysteresis in cascade systems [50].

\section{Conflict of Interests}

The author declares that there is no conflict of interests regarding the publication of this paper.

\section{Acknowledgment}

The author gratefully acknowledges financial support from the Slovak Scientific Grant Agency (VEGA).

\section{References}

[1] M. A. Krasnosel'skii and A. V. Pokrovskii, Systems with Hysteresis, Nauka, Moscow, Russia, 1983, (Russian).

[2] I. D. Mayergoyz, Mathematical Models of Hysteresis, Springer, New York, NY, USA, 1991.

[3] X. Chen, T. Hisayama, and C.-Y. Su, "Pseudo-inverse-based adaptive control for uncertain discrete time systems preceded by hysteresis," Automatica, vol. 45, no. 2, pp. 469-476, 2009.

[4] X. Chen and T. Ozaki, "Adaptive control for plants in the presence of actuator and sensor uncertain hysteresis," IEEE Transactions on Automatic Control, vol. 56, no. 1, pp. 171-177, 2011.

[5] X. Tan and J. S. Baras, "Modeling and control of hysteresis in magnetostrictive actuators," Automatica, vol. 40, no. 9, pp. 1469-1480, 2004.

[6] Y. Cao and X. B. Chen, "A novel discrete ARMA-based model for piezoelectric actuator hysteresis," IEEE/ASME Transactions on Mechatronics, vol. 17, no. 4, pp. 737-744, 2012.

[7] V. Kalaš, L. Jurišica, M. Žalman et al., Nonlinear and Numerical Servosystems, Alfa/SNTL, Bratislava, Slovakia, 1985 (Slovak).

[8] J. W. Macki, P. Nistri, and P. Zecca, "Mathematical models for hysteresis," SIAM Review, vol. 35, no. 1, pp. 94-123, 1993.

[9] E.-W. Bai, "Identification of linear systems with hard input nonlinearities of known structure," Automatica, vol. 38, no. 5, pp. 853-860, 2002.

[10] F. Ikhouane and J. Rodellar, "On the hysteretic Bouc-Wen model. part I. Forced limit cycle characterization," Nonlinear Dynamics, vol. 42, no. 1, pp. 63-78, 2005.

[11] F. Ikhouane and J. Rodellar, "On the hysteretic Bouc-Wen model. part II. Robust parametric identification," Nonlinear Dynamics, vol. 42, no. 1, pp. 79-95, 2005.

[12] J. H. Oh and D. S. Bernstein, "Semilinear Duhem model for rateindependent and rate-dependent hysteresis," IEEE Transactions on Automatic Control, vol. 50, no. 5, pp. 631-645, 2005.

[13] Y. Rochdi, F. Giri, F. Ikhouane, F. Z. Chaoui, and J. Rodellar, "Parametric identification of nonlinear hysteretic systems," Nonlinear Dynamics, vol. 58, no. 1-2, pp. 393-404, 2009.

[14] L. O. Chua and S. C. Bass, "A generalized hysteresis model," IEEE Transactions on Circuit Theory, vol. 19, no. 1, pp. 36-48, 1972.
[15] B. D. Coleman and M. L. Hodgdon, "A constitutive relation for rate-independent hysteresis in ferromagnetically soft materials," International Journal of Engineering Science, vol. 24, no. 6, pp. 897-919, 1986.

[16] M. L. Hodgdon, "Applications of a theory of ferromagnetic hysteresis," IEEE Transactions on Magnetics, vol. 24, no. 1, pp. 218-221, 1988.

[17] M. L. Hodgdon, "Mathematical theory and calculations of magnetic hysteresis curves," IEEE Transactions on Magnetics, vol. 24, no. 6, pp. 3120-3122, 1988.

[18] R. Banning, W. L. de Koning, H. J. Adriaens, and R. K. Koops, "State-space analysis and identification for a class of hysteretic systems," Automatica, vol. 37, no. 12, pp. 1883-1892, 2001.

[19] R. Merry, M. Uyanik, R. van de Molengraft, R. Koops, M. van Veghel, and M. Steinbuch, "Identification, control and hysteresis compensation of a 3 DOF metrological AFM," Asian Journal of Control, vol. 11, no. 2, pp. 130-143, 2009.

[20] C.-Y. Su, Y. Stepanenko, J. Svoboda, and T. P. Leung, "Robust adaptive control of a class of nonlinear systems with unknown backlash-like hysteresis," IEEE Transactions on Automatic Control, vol. 45, no. 12, pp. 2427-2432, 2000.

[21] P. J. van Bree, C. M. M. van Lierop, and P. P. J. van den Bosch, "Control-oriented hysteresis models for magnetic electron lenses," IEEE Transactions on Magnetics, vol. 45, no. 11, pp. 5235-5238, 2009.

[22] J. Zhou, C. Wen, and Y. Zhang, "Adaptive backstepping control of a class of uncertain nonlinear systems with unknown backlash-like hysteresis," IEEE Transactions on Automatic Control, vol. 49, no. 10, pp. 1751-1757, 2004.

[23] J. Vörös, "Modeling and parameter identification of systems with multisegment piecewise-linear characteristics," IEEE Transactions on Automatic Control, vol. 47, no. 1, pp. 184-188, 2002.

[24] K. Deng and F. Ding, "Newton iterative identification method for an input nonlinear finite impulse response system with moving average noise using the key variables separation technique," Nonlinear Dynamics, vol. 76, no. 2, pp. 1195-1202, 2014.

[25] F. Ding and T. Chen, "Identification of Hammerstein nonlinear ARMAX systems," Automatica, vol. 41, no. 9, pp. 1479-1489, 2005.

[26] P. Crama, J. Schoukens, and R. Pintelon, "Generation of enhanced initial estimates for Hammerstein systems," Automatica, vol. 40, no. 7, pp. 1269-1273, 2004.

[27] F. Ding, X. P. Liu, and G. Liu, "Identification methods for Hammerstein nonlinear systems," Digital Signal Processing, vol. 21, no. 2, pp. 215-238, 2011.

[28] J. Li and F. Ding, "Maximum likelihood stochastic gradient estimation for Hammerstein systems with colored noise based on the key term separation technique," Computers and Mathematics with Applications, vol. 62, no. 11, pp. 4170-4177, 2011.

[29] J. Chen, X. Wang, and R. Ding, "Gradient based estimation algorithm for Hammerstein systems with saturation and deadzone nonlinearities," Applied Mathematical Modelling, vol. 36, no. 1, pp. 238-243, 2012.

[30] X. Lv and X. Ren, "Non-iterative identification and model following control of Hammerstein systems with asymmetric dead-zone non-linearities," IET Control Theory \& Applications, vol. 6, no. 1, pp. 84-89, 2012.

[31] R. Pupeikis, "On the identification of Hammerstein systems having saturation-like functions with positive slopes," Informatica, vol. 17, no. 1, pp. 55-68, 2006. 
[32] G. Dolanc and S. Strmčnik, "Identification of nonlinear systems using a piecewise-linear Hammerstein model," Systems \& Control Letters, vol. 54, no. 2, pp. 145-158, 2005.

[33] F. Wang, K. Xing, and X. Xu, "Parameter estimation of piecewise Hammerstein systems," Transactions of the Institute of Measurement and Control, vol. 36, no. 8, pp. 1024-1032, 2014.

[34] H. F. Zou, "Identification of Hammerstein systems with twosegment preload nonlinearity based on the gradient search," Applied Mechanics and Materials, vol. 651-653, pp. 2314-2317, 2014.

[35] F. Giri, Y. Rochdi, F. Z. Chaoui, and A. Brouri, "Identification of Hammerstein systems in presence of hysteresis-backlash and hysteresis-relay nonlinearities," Automatica, vol. 44, no. 3, pp. 767-775, 2008.

[36] H. Chen, Y. Xiao, and F. Ding, "Hierarchical gradient parameter estimation algorithm for Hammerstein nonlinear systems using the key term separation principle," Applied Mathematics and Computation, vol. 247, pp. 1202-1210, 2014.

[37] Q. Shen and F. Ding, "Iterative estimation methods for Hammerstein controlled autoregressive moving average systems based on the key-term separation principle," Nonlinear Dynamics, vol. 75, no. 4, pp. 709-716, 2014.

[38] D. Wang, F. Ding, and Y. Chu, "Data filtering based recursive least squares algorithm for Hammerstein systems using the keyterm separation principle," Information Sciences, vol. 222, pp. 203-212, 2013.

[39] E.-W. Bai and K.-S. Chan, "Identification of an additive nonlinear system and its applications in generalized Hammerstein models," Automatica, vol. 44, no. 2, pp. 430-436, 2008.

[40] Z. Szabó, G. Szederkényi, P. Gáspár, I. Varga, K. M. Hangos, and J. Bokor, "Identification and dynamic inversion-based control of a pressurizer at the Paks NPP," Control Engineering Practice, vol. 18, no. 5, pp. 554-565, 2010.

[41] X. Li, L. Zhou, R. Ding, and J. Sheng, "Recursive least-squares estimation for Hammerstein nonlinear systems with nonuniform sampling," Mathematical Problems in Engineering, vol. 2013, Article ID 240929, 8 pages, 2013.

[42] C. Yu, C. Zhang, and L. Xie, "A new deterministic identification approach to Hammerstein systems," IEEE Transactions on Signal Processing, vol. 62, no. 1, pp. 131-140, 2014.

[43] Z. Hasiewicz and G. Mzyk, "Combined parametric-nonparametric identification of Hammerstein systems," IEEE Transactions on Automatic Control, vol. 49, no. 8, pp. 1370-1375, 2004.

[44] W. Zhao, W. X. Zheng, and E.-W. Bai, "A recursive local linear estimator for identification of nonlinear ARX systems: asymptotical convergence and applications," IEEE Transactions on Automatic Control, vol. 58, no. 12, pp. 3054-3069, 2013.

[45] H. N. Al-Duwaish, "Identification of hammerstein models with known nonlinearity structure using particle swarm optimization," Arabian Journal for Science and Engineering, vol. 36, no. 7, pp. 1269-1276, 2011.

[46] A. Janczak, "Neural network approach for identification of Hammerstein systems," International Journal of Control, vol. 76, no. 17, pp. 1749-1766, 2003.

[47] J. Vörös, "Parameter identification of discontinuous Hammerstein systems," Automatica, vol. 33, no. 6, pp. 1141-1146, 1997.

[48] J. Vörös, "Parametric identification of systems with general backlash," Informatica, vol. 23, no. 2, pp. 283-298, 2012.

[49] F. Ding, Y. Wang, and J. Ding, "Recursive least squares parameter identification algorithms for systems with colored noise using the filtering technique and the auxilary model," Digital Signal Processing, vol. 37, pp. 100-108, 2015.

[50] J. Vörös, "Modeling and identification of hysteresis using special forms of the Coleman-Hodgdon model," Journal of Electrical Engineering, vol. 60, no. 2, pp. 100-105, 2009. 


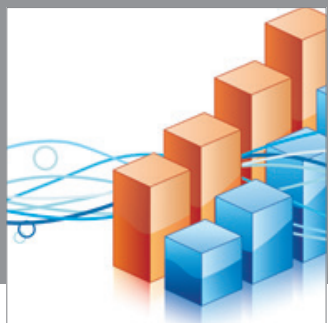

Advances in

Operations Research

mansans

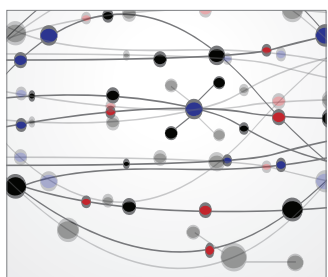

The Scientific World Journal
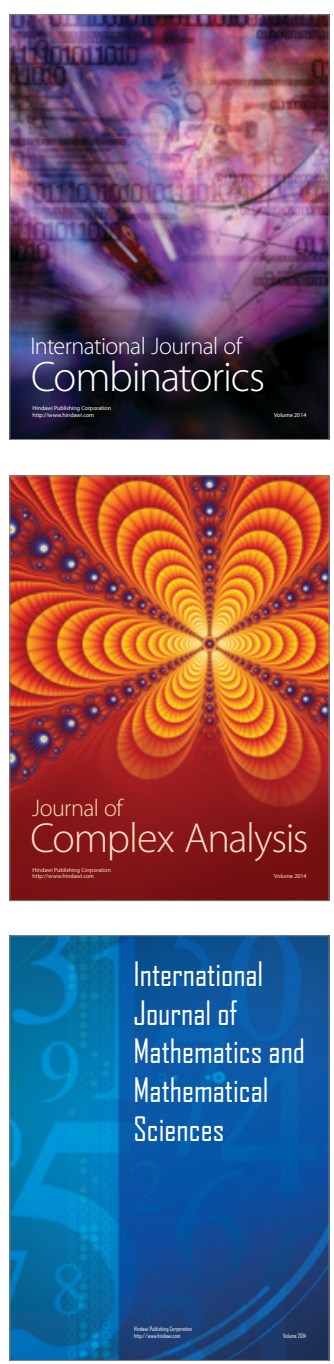
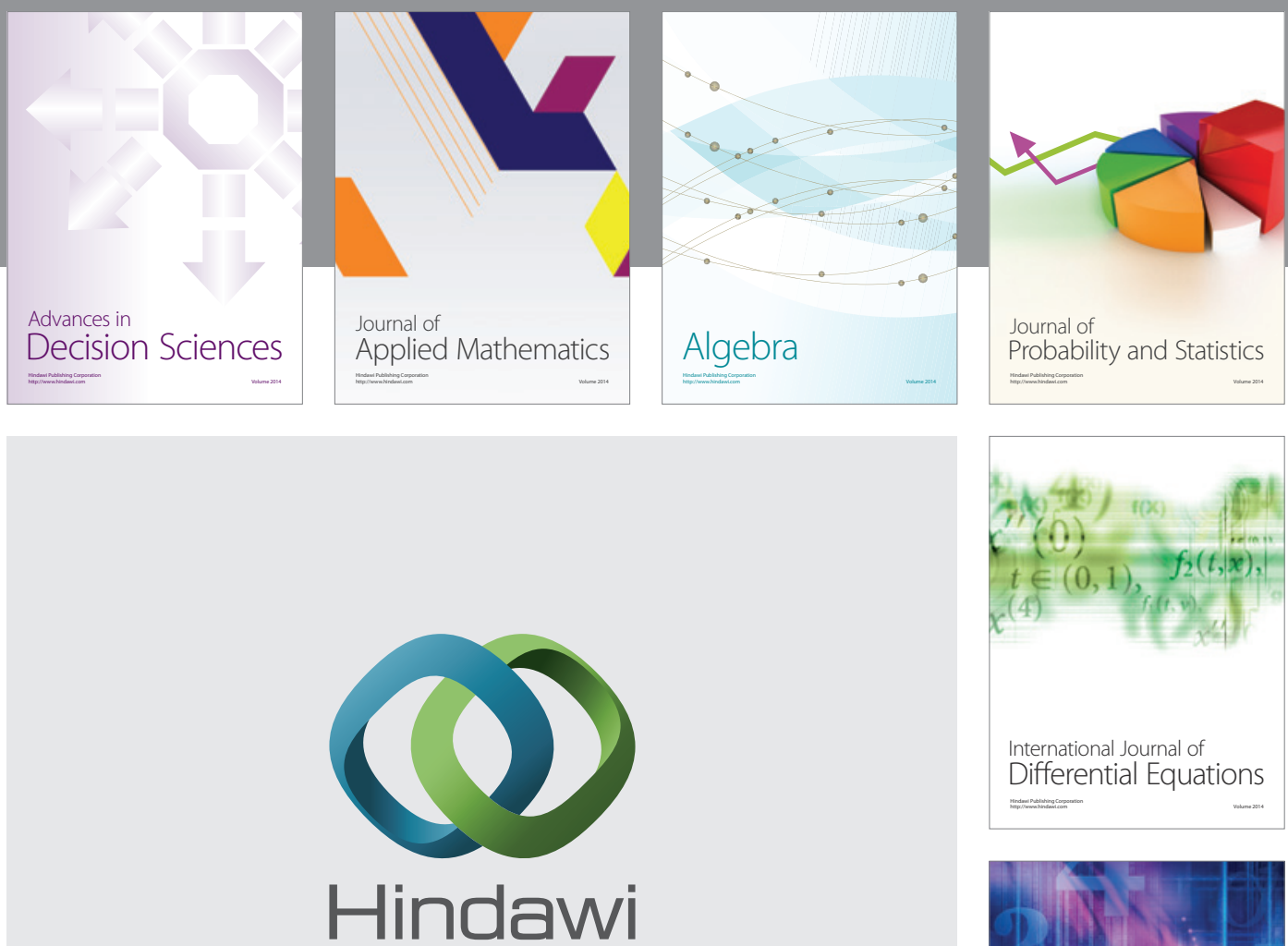

Submit your manuscripts at http://www.hindawi.com
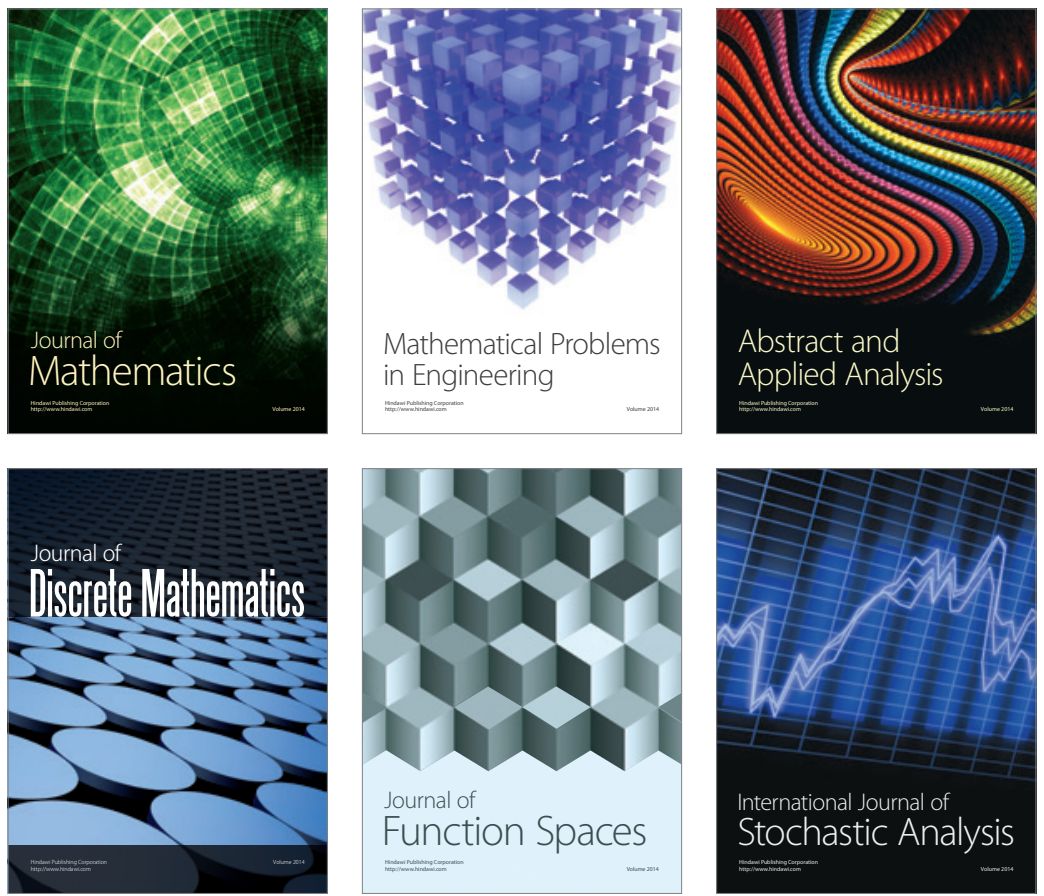

Journal of

Function Spaces

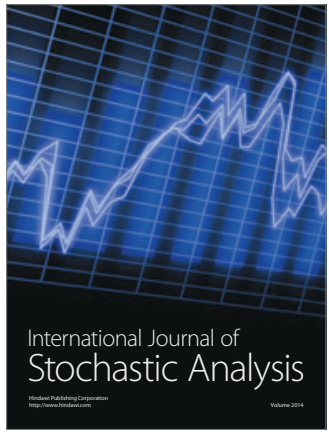

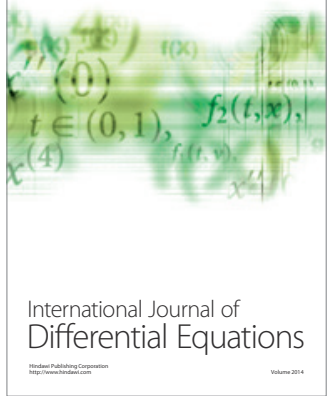
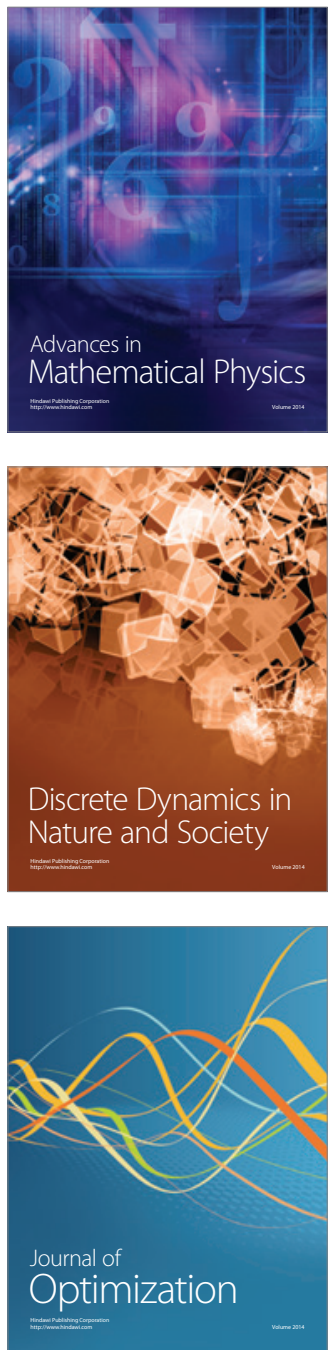